\title{
Manejo da irrigação em pastagem irrigada por pivô-central
}

\author{
Alexandre C. Xavier ${ }^{1}$, Rubens D. Coelho ${ }^{2}$, Leandro F. Lourenço ${ }^{3}$ \& Ronalton E. Machado ${ }^{4}$ \\ 1 UFES/CCA/DER. CP 16, CEP 29500-000, Alegre, ES. Fone (28) 3552-1400. E-mail: xavier@cca.ufes.br (Foto) \\ 2 USP/ESALQ, Depto. de Engenharia Rural, CP 9 - CEP:13418-900 - Piracicaba, SP. Fone: (19) 3429-4217 ramal 223 \\ E-mail: rdcoelho@esalq.usp.br \\ 3 Bolsista CAPES. Fone: (19) 3429-4217 ramal 223. E-mail: Iflouren@carpa.ciagri.usp.br \\ 4 USP/ESALQ. Fone (19) 3429-4143 ramal 208. E-mail: rmachad@ig.com.br
}

Protocolo $39-25 / 2 / 2003$ - Aprovado em 6/2/2004

\begin{abstract}
Resumo: A aplicação de lâminas de irrigação em pastagem irrigada sob pivô-central é, de maneira geral, realizada sem um critério técnico pertinente ao sistema, pois se deve considerar que para um mesmo período a pastagem se encontra em diferentes estádios de desenvolvimento em cada parcela, apresentando taxas de evapotranspiração diferenciadas dentro da área irrigada; todavia, usualmente se aplica uma única lâmina para toda a área. Neste trabalho foi desenvolvido um modelo para aplicação de lâminas de irrigação distinta para cada parcela do pivô o qual, de modo geral, considera: i) a capacidade do pivô-central de aplicar lâminas distintas na área; ii) o nível de desenvolvimento da cultura em cada parcela; iii) o período de retorno do gado a determinada parcela (ciclo de pastejo); e iv) o potencial de desenvolvimento da pastagem de certa região. Para modelar o coeficiente de cultura $(\mathrm{Kc})$ foram utilizadas duas metodologias, a primeira com taxa de variação do Kc constante com o número de dias em que a parcela está em descanso (k), e a segunda, com taxa de variação do Kc na forma senoidal com k. O modelo foi aplicado para pastagens hipotéticas nas regiões de Piracicaba e Pereira Barreto, para avaliação e, como resultado, observou-se que o modelo se mostrou sensível ao nível de desenvolvimento de cada parcela e às condições de variação do clima de cada região.
\end{abstract}

Palavras-chave: capim Tanzânia, manejo da água, coeficiente de cultura

\section{Irrigated pasture: Water management under center pivot irrigation}

\begin{abstract}
The application of irrigation depths in irrigated pasture under center pivot machines, in a general way, is accomplished without a pertinent technical criterion, because it should be considered that for any time period, the pasture plots are at different development stages (rotary pasture), presenting different evapotranspiration rates inside the irrigated area. Furthermore, farmers usually apply a single irrigation depth for the whole area. In this study a model was developed for the application of different irrigation depths in each portion of the pivot (pizza shape plots). The model proposed in this study considers: i) the capacity of the pivot-center to apply different water depths in the area; ii) development stage of crop in each plot iii) cattle return period for grazing in-determined plot (pasture cycle fixed); and iv) potential of pasture development during the agronomic year. To compute the crop coefficient $(\mathrm{Kc})$ variable in the model, two methodologies were used: a) constant variation rate of $\mathrm{Kc}$, according to the resting days the portion is submitted and $\mathrm{b}$ ) sinusoidal variation rate of $\mathrm{Kc}$, according to the resting period. The model was applied for hypothetical pastures conditions under rural areas of Piracicaba and Pereira Barreto municipalities, in the State of São Paulo, Brazil. The model's sensitivity for the irrigation depths applied was observed for the different stages of Tanzania grass development under the center-pivot, according to the variable climatic conditions of each region.
\end{abstract}

Key words: Tanzânia grass, irrigation management, crop coefficient 


\section{INTRODUÇÃO}

Pecuaristas de várias regiões do Brasil vêm adotando o sistema de irrigação por aspersão tipo pivô-central, em pastagens formadas por gramíneas do gênero Panicum (Capim Tanzânia e Monbaça) e Brachiaria (Capim Braquiária), aliado ao uso intensivo de adubações de reposição (N, P e K). Estas áreas são manejadas de forma rotacionada, com ciclo variando entre 28 a 36 dias, sendo o consumo do capim diretamente por pastejo ou através do armazenamento em silagem, utilizada para o confinamento. Todavia, só a irrigação e nutrientes não são condições suficientes para o aumento significativo da produção mas, sim, a associação destas com condições climatológicas adequadas, por exemplo, temperatura adequada para o desenvolvimento (Müller et al., 2002).

A utilização da irrigação em gramíneas, conduzidas sob pastejo rotacionado, é uma técnica mais complexa do que se imaginava inicialmente. Segundo Lourenço et al. (2001) a evapotranspiração do capim Tanzânia posterior ao corte foi de 4 a 5 vezes inferior àquela apresentada ao final do ciclo. Tal resultado foi associado ao índice de área foliar (IAF) de 1,0 a $1,5 \log$ após o corte e de 4,0 a 5,5 ao final do ciclo. Os mesmos autores verificaram, também, que o coeficiente de cultura $(\mathrm{Kc})$ variou de 0,30 a 0,40 na fase inicial de desenvolvimento e de 1,10 a 1,40 na fase final, para pastagem com ciclo de 36 dias. Tal variação do Kc influenciou diretamente no manejo de irrigação. Para exemplificar o fato, seja o sistema de pastagem rotacionada em pivô-central apresentada na Figura 1 com a rotação dos animais ocorrendo no sentido anti-horário. A parcela de número três é suposta como sendo a parcela em que os animais se encontram em pastejo; logo, seu IAF será mínimo ao final do pastejo nesta área, implicando em um coeficiente de cultivo mínimo $\left(\mathrm{Kc}_{\min }\right)$, apresentando necessidade de irrigação também mínima; já a parcela quatro se encontra em pré-pastejo com o número máximo de dias de descanso $\left(\mathrm{k}_{\mathrm{pmax}}\right)$ de acordo com o ciclo local e, daí, apresentará um desenvolvimento $\left(\mathrm{IAF}_{\max }\right)$ maior, implicando em maior coeficiente de cultivo $\left(\mathrm{Kc}_{\max }\right)$ e na necessidade de maior lâmina de irrigação. Verifica-se, seguindose o raciocínio acima, que as parcelas posteriores a quatro $(\mathrm{p}=5,6, \ldots, \mathrm{n}, 1,2)$ apresentarão um número menor de dias de descanso quando comparadas com a parcela 4 , ou seja, menores IAF, Kc e taxas de evapotranspiração, quando comparada com a parcela quatro.

Todavia, na prática se verifica que é aplicada uma lâmina de irrigação uniforme em toda a área do pivô, sem que haja discriminação do grau de desenvolvimento de cada parcela, podendo acarretar perdas/excesso de água e energia, caso o solo não consiga atuar como reservatório de alta eficiência.

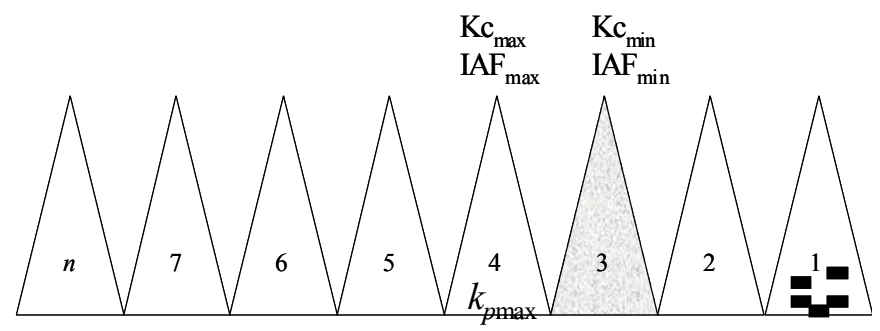

Figura 1. Modelo linear para lâmina de irrigação
Desta forma, o objetivo do presente trabalho foi desenvolver um modelo para estimativa da lâmina de irrigação necessária para suprir as necessidades de água do capim Tanzânia (Panicum maximun Jacq) de acordo com a determinação do Kc de uma parcela de controle e se considerando as condições climatológicas locais.

\section{MATERIAL E MÉTODOS}

Assume-se que o pivô-central tem capacidade finita de aplicar diferentes lâminas na área a ser irrigada podendo-se, então, dividir a área do pivô em p parcelas (Figura 2). Os animais pastejam no sentido anti-horário, retornando a determinada parcela depois de certo número de dias de descanso $\left(\mathrm{k}_{\mathrm{pmax}}\right)$ conforme ciclo estabelecido para a região. Segundo Lourenço et al. (2001) a necessidade de irrigação de cada parcela varia de acordo com o estado de desenvolvimento da pastagem que, por outro lado, é dependente do número de dias que se encontra em descanso após o pastejo (k). No modelo se considera $\mathrm{p}=1$ como sendo a parcela de controle e que a lâmina a ser irrigada nesta parcela será obtida com auxílio de dados de tensão da água no solo, coletados através de baterias de tensiômetros, a diferentes profundidades para um monitoramento da umidade no perfil do solo.

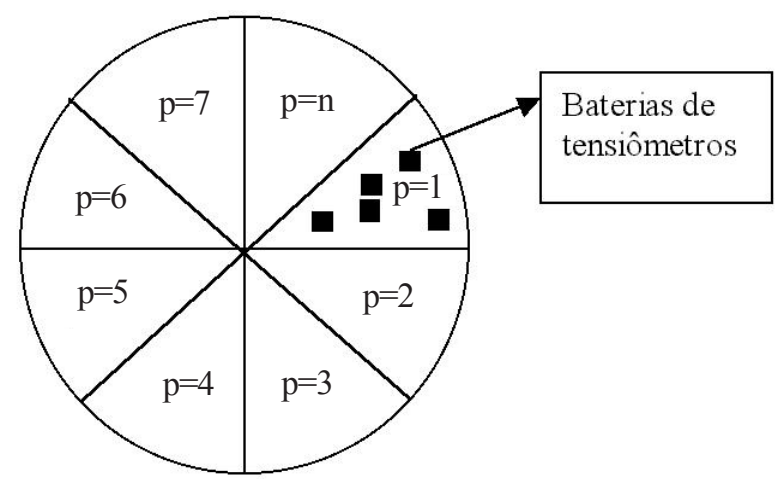

Figura 2. Esquema do pivô-central para áreas de pastagem

O modelo que será apresentado tem, como suposição para fins práticos, uma relação direta entre lâmina de irrigação a ser aplicada e o seu respectivo coeficiente da cultura $(\mathrm{Kc}) \mathrm{e}$, desta forma, com o número de dias em que cada parcela está em descanso (k).

\section{Lâmina de irrigação na parcela de controle monitorada por tensiometria}

A lâmina bruta total a ser aplicada na parcela $1\left(\mathrm{~L}_{1}\right)$ é calculada pela equação:

$$
\mathrm{L}_{1}=\frac{1}{\eta} \sum_{j=1}^{h}\left(\theta_{\mathrm{CC}_{j}}-\theta_{\mathrm{obs}_{j}}\right) \mathrm{Z}_{j},
$$

em que h é o número de camadas monitoradas e $\eta$ é a eficiência do sistema de irrigação; $\theta_{\mathrm{CC}}$ é a umidade da capacidade de campo, $\mathrm{cm}^{3} \mathrm{~cm}^{-3} ; \theta_{\text {obs }}$ é a umidade observada no solo, $\mathrm{cm}^{3} \mathrm{~cm}^{-3}$; e Z a altura da camada a ser considerada, $\mathrm{mm}$. $\theta_{\text {obs }}$ será calculada por meio da seguinte equação (van Genuchten, 1980): 


$$
\theta_{\text {obs }_{j}}=\theta_{\mathrm{r}}+\frac{\theta_{\mathrm{s}}-\theta_{\mathrm{r}}}{\left.1+\left(\alpha\left|\Psi_{j}\right|\right)^{\mathrm{n}}\right]^{\mathrm{m}}}
$$

em que: $\theta_{\mathrm{s}}$ é a umidade de saturação do solo, $\mathrm{cm}^{3} \mathrm{~cm}^{-3} ; \theta_{\mathrm{r}}$ é a umidade residual do solo, $\mathrm{cm}^{3} \mathrm{~cm}^{-3} ; \alpha, \mathrm{n}$ e m são os parâmetros; sendo

$$
\Psi_{j}=\frac{1}{l} \sum_{i=1}^{l} \Psi_{i}
$$

em que $\Psi_{j}$ é a tensão média lida na superfície do solo (cm.c.a) observada das l-ésimas medidas dos tensiômetros $(\Psi)$ na profundidade $\mathrm{j}$.

Assumindo-se que a taxa de variação da lâmina de irrigação para uma parcela $\left(\mathrm{L}_{p}\right)$ com o seu respectivo $\mathrm{Kc}\left(\mathrm{Kc}_{p}\right)$ é constante $(\varepsilon)$, tem-se, após integração, que

$$
\mathrm{L}_{p}(\mathrm{Kc})=\varepsilon \mathrm{Kc}_{p}+C_{1}
$$

em que $C_{1}$ é uma constante.

Na parcela de controle tanto a lâmina a ser aplicada como o coeficiente de cultura são conhecidos, $\log 0 \mathrm{~L}\left(\mathrm{Kc}_{1}\right)=\mathrm{L}_{1}$. Segue, também, que quando Kc for zero, a lâmina a ser irrigada será zero; então, $\mathrm{L}(0)=0$; desta forma a Eq. 4 pode ser reescrita como:

$$
\mathrm{L}_{p}\left(\mathrm{Kc}_{p}\right)=\frac{\mathrm{L}_{1}}{\mathrm{Kc}_{1}} \mathrm{Kc}_{p}
$$

\section{Estimativa do coeficiente de cultura para cada parcela}

$\mathrm{O}$ coeficiente de cultura para cada parcela $\left(\mathrm{Kc}_{p}\right)$ será modelado de duas maneiras devido a duas situações hipotéticas para o comportamento do $\mathrm{Kc}$ verificado por Lourenço et al. (2001):

a. Modelo linear para variação do $\mathbf{K c}_{\mathbf{p}}$ : Este modelo pressupõe que a taxa de variação dos $\mathrm{Kc}_{p}$ s é constante $(\phi)$ com a variação $\mathrm{k}_{\mathrm{p}}$ ou seja,

$$
\frac{\mathrm{dKc}_{\mathrm{p}}}{\mathrm{dk}_{\mathrm{p}}}=\phi
$$

integrando esta equação,

$$
\mathrm{Kc}_{p}\left(k_{p}\right)=\phi k_{p}+C_{2}
$$

Para resolver o problema de valor inicial, considera-se que determinada parcela (p) terá, no dia zero (início do pastejo) um consumo mínimo de água, a que se denomina coeficiente de cultura mínimo $\left(\mathrm{Kc}_{\min }\right)$; desta forma, $\mathrm{Kc}(0)=\mathrm{Kc}_{\min }$. Também, de acordo com as condições climatológicas do respectivo período analisado para o máximo número de dias de descanso (prépastejo) da parcela $\mathrm{p}$, o Kc será máximo; isto é, $\mathrm{Kc}\left(k_{p \max }\right)=\mathrm{Kc}_{\max }$. A Eq. 7 pode, então, ser reescrita da seguinte forma:

$$
\mathrm{Kc}_{p}\left(k_{p}\right)=\frac{\left(\mathrm{Kc}_{\max }-\mathrm{Kc}_{\min }\right)}{k_{p \max }} k_{p}+\mathrm{Kc}_{\text {min }}
$$

b. Modelo senoidal para variação do $\mathrm{Kc}_{\mathrm{p}}$ : Para o modelo senoidal diz-se que a taxa de variação do Kc pode não ser constante para os números de dias de descanso de determinada parcela, mas ela será da forma senoidal, com uma taxa de variação inicial $\left(\mathrm{dKc}_{\mathrm{p}}(0) / \mathrm{dy}=\delta\right)$ crescente até um máximo, que ocorreria na metade dos dias de descanso; na segunda metade dos dias de descanso ocorre uma queda na taxa de variação do Kc; assim sendo, $\mathrm{y}=\mathrm{f}\left(\mathrm{k}_{\mathrm{p}}\right)=\pi \mathrm{k}_{\mathrm{p}} / \mathrm{k}_{\mathrm{pmax}}$, pode-se escrever:

$$
\frac{d \mathrm{Kc}_{\mathrm{p}}}{\mathrm{dy}}=\delta+\varphi \operatorname{sen}(\mathrm{y})
$$

integrando esta equação obtém-se

$$
\mathrm{Kc}_{\mathrm{p}}\left(\mathrm{k}_{\mathrm{p}}\right)=\delta\left(\frac{\pi \mathrm{k}_{\mathrm{p}}}{\mathrm{k}_{\mathrm{pmax}}}\right)-\varphi \cos \left(\frac{\pi \mathrm{k}_{\mathrm{p}}}{\mathrm{k}_{\mathrm{pmax}}}\right)+\mathrm{C}_{3}
$$

Resolvendo o problema de valor inicial com as condições conforme mostradas no modelo linear $\left(\mathrm{Kc}_{\mathrm{p}}(0)=\mathrm{Kc}_{\min } \mathrm{e}\right.$ $\left.\mathrm{Kc}_{\mathrm{p}}\left(k_{p \max }\right)=\mathrm{Kc}_{\text {max }}\right)$, tem-se que:

$$
\begin{aligned}
\mathrm{Kc}_{p}\left(k_{p}\right)= & \delta \frac{\pi k_{p}}{k_{p \max }}-\frac{\mathrm{Kc}_{\text {max }}-\mathrm{Kc}_{\text {min }}-\delta \pi}{2} \cos \left(\frac{\pi k_{p}}{k_{p \max }}\right)+ \\
& \frac{\mathrm{Kc}_{\text {max }}+\mathrm{Kc}_{\text {min }}-\delta \pi}{2}
\end{aligned}
$$

\section{Cálculo do $\mathrm{Kc}_{\text {max }}$ ao longo do ano}

A produção de matéria seca em pastagem é influenciada pela temperatura do ar (Müller et al., 2002; Lourenço et al., 2001). Villa Nova et al. (1983) propuseram utilizar a Unidade Fototérmica (UF) como maneira de mensurar a energia em determinada época. Para isto, sugerem a equação:

$$
\mathrm{UF}=\frac{\left(\frac{\mathrm{n}}{2} \overline{\mathrm{GD}}\right)^{\frac{\mathrm{Nf}}{\mathrm{Ni}}+1}}{\frac{\mathrm{Nf}}{\mathrm{Ni}}+1}
$$

em que: $\overline{\mathrm{GD}}$ são os graus-dia médios do período de $n$ dias; Nf, o fotoperíodo (horas e décimos) no final do período de crescimento, e $\mathrm{Ni}$ o fotoperíodo, no início do período de crescimento.

Para modelar o $\mathrm{Kc}_{\text {max, }}$ com base na UF, utilizar-se-á um modelo logístico, com capacidade de tolerância para o coeficiente de cultura. Neste modelo, a capacidade de aumento de Kc diminui com o aumento da UF. Desta forma, pode-se escrever:

$$
\frac{1}{\mathrm{Kc}} \frac{\mathrm{dKc}}{\mathrm{dUF}}=\tau\left(1-\frac{\mathrm{Kc}}{\mathrm{T}}\right)
$$


em que: T é a capacidade de tolerância para o Kc e $\tau$ é uma constante. Nota-se, pela Eq. 13, que quando Kc é pequeno comparado com T, então Kc/T será próximo de zero; logo, dKc/ $\mathrm{dUF} \approx \tau \mathrm{Kc}$, ou seja, Kc tende a crescer de forma exponencial na direção de $\mathrm{T}$; no entanto, se $\mathrm{Kc} \rightarrow \mathrm{T}$ (coeficiente de cultura tende à sua capacidade de tolerância), então $\mathrm{Kc} / \mathrm{T} \rightarrow 1$; logo, $\mathrm{dKc} / \mathrm{dUF} \rightarrow 0$, representando o equilíbrio com a capacidade do sistema, tendendo a permanecer estável. Resolvendo a Eq.13, tem-se:

$$
\mathrm{Kc}(\mathrm{UF})=\frac{\mathrm{T}}{1+\mathrm{A} e^{-\tau \mathrm{UF}}}
$$

sendo

$$
\mathrm{A}=\frac{\mathrm{T}-\mathrm{Kc}_{0}}{\mathrm{Kc}_{0}}
$$

A capacidade máxima de tolerância de Kc para o sistema será de 1,55 e o Kc igual a 0,35 (Lourenço et al., 2001). Também, segundo Mello et al. (2001), Kc(6000) =1,00. Substituindo-se esses valores na Eq. 15, tem-se

$$
\mathrm{Kc}(\mathrm{UF})=\frac{1,55}{1+3,42 e^{-0,0003045 \mathrm{UF}}}
$$

O modelo logístico pode ser utilizado para se determinar o Kc máximo diário em qualquer região para capim Tanzânia, desde que se tenha as séries de temperaturas mensais (utilizadas para calcular graus-dias) e a quantidade de dias em que a parcela permanece em descanso $\left(\mathrm{k}_{\mathrm{pmax}}\right)$.

\section{Dias de descanso de cada parcela}

Tem-se, agora, o problema da determinação do número de dias de descanso para cada parcela $\left(\mathrm{k}_{\mathrm{p}}\right)$ do pivô-central. A parcela de controle é a única em que o número de dias de descanso é acompanhado $\left(\mathrm{k}_{1}\right)$; a partir da segunda parcela, o número de dias de descanso será determinado por:

$$
\begin{gathered}
\mathrm{k}_{\mathrm{p}}=\mathrm{k}_{\mathrm{p}-1}-\mathrm{k}_{\mathrm{p} \max } / \mathrm{p}, \text { se } \mathrm{k}_{\mathrm{p}-1}-\mathrm{k}_{\mathrm{pmax}} / \mathrm{p}>0, \text { ou } \\
\mathrm{k}_{\mathrm{p}}=\mathrm{k}_{\mathrm{pmax}}-\left(\mathrm{k}_{\mathrm{p} \max } / \mathrm{p}-\mathrm{k}_{\mathrm{p}-1}\right), \quad \text { se } \mathrm{k}_{\mathrm{p}-1}-\mathrm{k}_{\mathrm{p} \max } / \mathrm{p} \leq 0 .
\end{gathered}
$$

sendo p o número da parcela em análise.

A porção " $k_{\text {pmax }} / p$ " é um fator de proporção para cada mudança de parcela, sendo que, quanto maior a quantidade de lâminas distintas que o pivô pode aplicar, considerando-se $\mathrm{k}_{\mathrm{pmax}}$ constante, menor será esta porção e mais fracionada a distribuição de aplicação da água na área. Em equipamentos pivô-central com painel digital é possível se estabelecer de 8 a 12 setores molhados por operação automática.

\section{Análise do modelo}

Será apresentada a aplicação do modelo proposto para as condições de pastagem irrigada por pivô-central nas regiões de Piracicaba $\left(21^{\circ} 42^{\prime \prime}\right.$ de latitude sul e $47^{\circ} 38^{\prime \prime}$ de longitude oeste) e Pereira Barreto $\left(20^{\circ} 41^{\prime \prime}\right.$ de latitude sul e $51^{\circ} 7 " \mathrm{de}$ longitude oeste), no Estado de São Paulo; essas duas regiões foram escolhidas por apresentarem condições climatológicas bem distintas permitindo, assim, que se avalie o comportamento do modelo em situações diferentes. O clima da região de Piracicaba é Cwa, subtropical úmido, conforme classificação de Köppen, com verão chuvoso e inverno seco, sendo de $1230 \mathrm{~mm}$ a precipitação média anual; as temperaturas médias mensais variam de $25,4^{\circ} \mathrm{C}$ no verão a $17,9^{\circ} \mathrm{C}$ no inverno, com média anual de 21,6 ${ }^{\circ} \mathrm{C}$ (LES-ESALQ/USP, 2002); já a região de Pereira Barreto apresenta precipitação média anual de $1128 \mathrm{~mm}$, com temperaturas máximas e mínimas de $26,8^{\circ} \mathrm{C}$ (fevereiro) e $21,2^{\circ} \mathrm{C}$ (junho) com média anual de $24,7^{\circ} \mathrm{C}$. A temperatura média mensal em Pereira Barreto é sempre superior à de Piracicaba, enquanto a precipitação em Piracicaba, na maioria dos meses é superior à de Pereira Barreto.

O manejo da irrigação por pivô-central em pastagem nas regiões de Piracicaba e Pereira Barreto, terá as seguintes características: i) pastagem com ciclo total de 36 dias; ii) irrigações realizadas em duas épocas, no dia primeiro de fevereiro $(\mathrm{J}=32, \mathrm{~J}$ é o dia juliano) e no dia primeiro de agosto $(\mathrm{J}=213)$ representando períodos com altas e baixas temperaturas, respectivamente; iii) irrigações baseadas em pivô-central com capacidade de se aplicar 12 lâminas distintas (p) na área, e iv) lâmina calculada para irrigação na parcela controle será conhecida, sendo $\mathrm{L}_{1}=10 \mathrm{~mm}$, encontrando-se no $16^{\circ}$ dia de descanso.

\section{RESULTADOS E DISCUSSÃO}

A Unidade Fitotérmica (UF) foi calculada para as duas regiões em estudo, com base em pastagem com o ciclo de 36 dias $\left(\mathrm{UF}_{36}\right)$, utilizando-se as séries históricas de temperatura média de cada região e localização geográfica. $\mathrm{O}$ comportamento geral da $\mathrm{UF}_{36}$ de cada região é apresentado na Figura 3. Para a região de Piracicaba, a máxima $U_{36}$ é observada no final de fevereiro, enquanto a mínima ocorre em julho; já para Pereira Barreto, a $\mathrm{UF}_{36}$ apresenta máxima em outubro e mínima no final de julho. Pode-se observar também que, em geral a $U_{36}$ de Pereira Barreto é maior que a de Piracicaba durante quase todo o ano, resultado principalmente de suas maiores temperaturas médias mensais, visto que as latitudes são próximas, o que influencia pouco nos respectivos fotoperíodos das regiões.

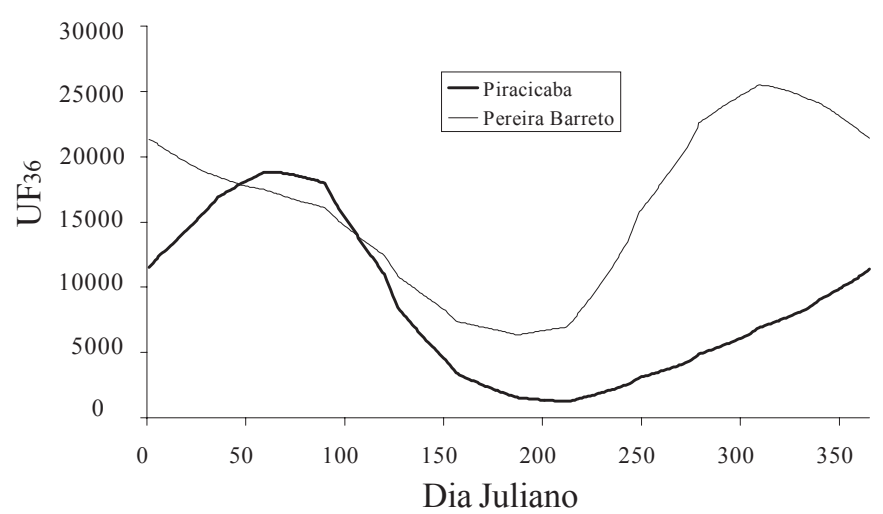

Figura 3. Comportamento da Unidade Fitotermica (UF) de 36 dias para as regiões em estudo 
Uma simplificação das curvas mostradas na Figura 3 por meio da utilização regressão múltipla de modelos polinomiais foi realizada, em que a $\mathrm{UF}_{36}$ e o dia Juliano são as variáveis dependente e independente, respectivamente; desta forma, tem-se uma estimativa da $\mathrm{UF}_{36}$ de maneira contínua no ano e não discreta. Verificou-se que o dia Juliano (J) utilizado nos modelos polinomiais do $4^{\circ}$ grau explicou mais de $95 \%$ da variação da $\mathrm{UF}_{36}$ para as duas regiões em estudo (Tabela 1).

Tabela 1. Modelos* para se estimar a $\mathrm{UF}_{36}$ para as regiões de Piracicaba e Pereira Barreto

Região Modelo polinomial para $\mathrm{UF}_{36}(J) \quad \mathrm{R}^{2}$

Piracicaba $8917,50+427,97 J-5,74 J^{2}+0,022 \mathcal{J}^{3}-2,74.10^{-5} J^{4} \quad 0,95$

P. Barreto $16957,00-104,65 J+2,48 J^{2}-0.029 \mathcal{P}^{3}-1,23.10^{-4} J^{4} \quad 0,97$

* Ambos os modelos foram significativos a $\mathrm{p}<0,0001$

Pode-se calcular, agora, $\mathrm{o} \mathrm{Kc}_{\text {max }}$ de maneira contínua, para o pastejo com 36 dias de ciclo, com base no modelo logístico para Piracicaba (Eq. 19) e para Pereira Barreto (Eq. 20).

$$
\begin{aligned}
& \mathrm{Kc}_{\text {max }, \mathrm{UF}} \mathrm{F}_{3(\text { (Pinacicaba) }}(\mathrm{J})=\frac{1,55}{1+3,42 \mathrm{e}^{\mathrm{T}}} \\
& \mathrm{Kc}_{\text {max,UF }} \mathrm{F}_{3(\mathrm{P} \text {. Barreto }}(\mathrm{J})=\frac{1,55}{1+3,42 \mathrm{e}^{\mathrm{T}}}
\end{aligned}
$$

sendo $T=-0,000304\left(16957,00-104,65 \mathrm{~J}+2,48 \mathrm{~J}^{2}-0,029 \mathrm{~J}^{3}-1,23.10^{-4} \mathrm{~J}^{4}\right.$

O modelo linear ou senoidal pode, então, ser utilizado para calcular o Kc de cada parcela em determinada época do ano. Considerando os $\mathrm{Kc}_{\max }$ obtido pela Eq. 19 e $20, \mathrm{Kc}_{\min }$ igual a 0,35 e $\delta=0,01$ (Lourenço et al., 2001), obtiveram-se as Eqs. 21 e 22 para Piracicaba, e as Eqs. 23 e 24, para Pereira Barreto, referentes aos modelos linear e senoidal, respectivamente.

$$
\mathrm{Kc}_{\mathrm{p}}\left(\mathrm{k}_{\mathrm{p}}, \mathrm{J}\right)=\frac{1}{36}\left(\mathrm{Kc}_{\text {max,UF } \mathrm{F}_{3(\text { (Priacicaba })}}-0,35\right) \mathrm{k}_{\mathrm{p}}+0,35
$$

$$
\begin{aligned}
& \mathrm{Kc}_{\mathrm{p}}\left(\mathrm{k}_{\mathrm{p}}, \mathrm{J}\right)= \\
& 0,01 \frac{\pi \mathrm{k}_{\mathrm{p}}}{36}-\frac{1}{2}\left(\mathrm{Kc}_{\max , \mathrm{UF}_{36(\text { Princiatab })}}-0,35-0,01 \pi\right) \cos \left(\frac{\pi \mathrm{k}_{\mathrm{p}}}{36}\right)+ \\
& \frac{1}{2}\left(\mathrm{Kc}_{\text {max }, \mathrm{UF}} \mathrm{F}_{3(\text { (Princicaba) }}+0,35-0,01 \pi\right)
\end{aligned}
$$

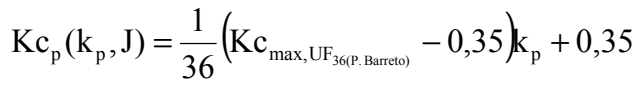

$$
\begin{aligned}
& \mathrm{Kc}_{\mathrm{p}}\left(\mathrm{k}_{\mathrm{p}}, \mathrm{J}\right)= \\
& 0,01 \frac{\pi \mathrm{k}_{\mathrm{p}}}{36}-\frac{1}{2}\left(\mathrm{Kc}_{\max , \mathrm{UF} \mathrm{F}_{\text {G(P. Barrcoo) }}}-0,35-0,01 \pi\right) \cos \left(\frac{\pi \mathrm{k}_{\mathrm{p}}}{36}\right)+ \\
& \frac{1}{2}\left(\mathrm{Kc}_{\max , \mathrm{UU} \mathrm{F}_{3(\mathrm{P} . \text {. Barreto }}}+0,35-0,01 \pi\right)
\end{aligned}
$$

Nas Figuras 4 e 5 pode-se observar o comportamento geral do Kc em qualquer dia Juliano para o ciclo da pastagem nas duas regiões em estudo, a partir dos modelos linear e senoidal, respectivamente constatando-se, sobretudo, o contraste entre a variação do Kc para as duas regiões em ambos os modelos. Na região de Piracicaba há queda considerável do Kc entre aproximadamente os $150^{\circ}$ e os $250^{\circ}$ dias Juliano ao longo de todo o ciclo da cultura, com valores próximos ao Kc mínimo $(0,35)$, enquanto para Pereira Barreto a queda do Kc é bem menor, cujo resultado é reflexo direto da baixa UF disponível para a cultura neste período, em Piracicaba, o mesmo não ocorrendo para a região de Pereira Barreto. De forma geral verifica-se, para a região de Piracicaba durante o inverno, uma necessidade menor de irrigação e se espera que a produtividade seja bastante afetada neste período, uma vez que a quantidade de energia disponível para o desenvolvimento da pastagem é limitada. Quanto à comparação entre os modelos (linear e senoidal), o modelo senoidal parece ser mais adequado ao

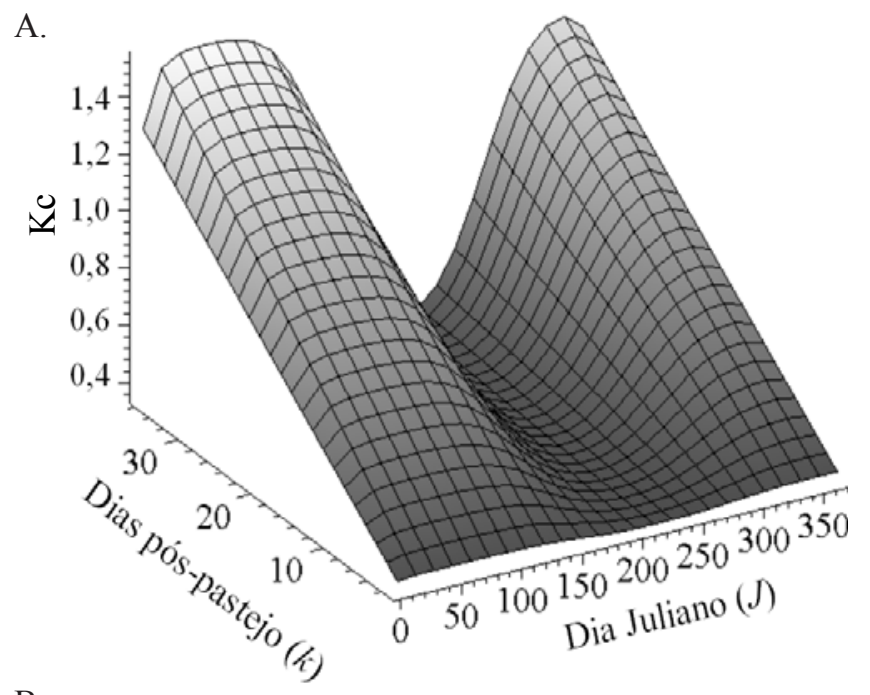

B.

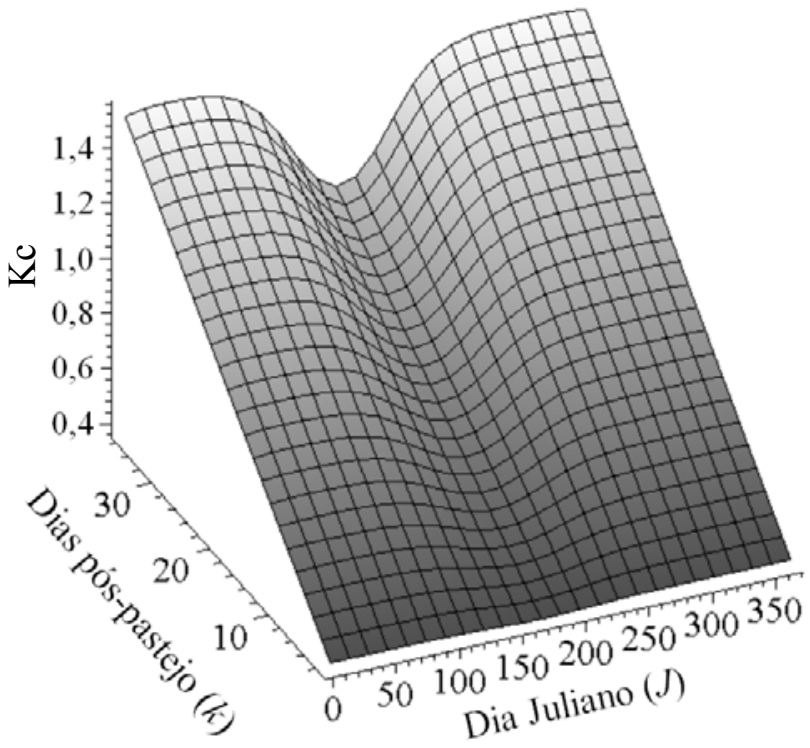

Figura 4. Kc calculado a partir do modelo linear em pastagem com ciclo completo de 36 dias durante um ano, para as regiões de Piracicaba (A) e Pereira Barreto (B) 
período de altas temperaturas, apresentando taxas de variação distintas para o Kc, com taxa de crescimento menor no início e no final do desenvolvimento, enquanto o modelo linear responderia melhor em período de baixas temperaturas,

A.

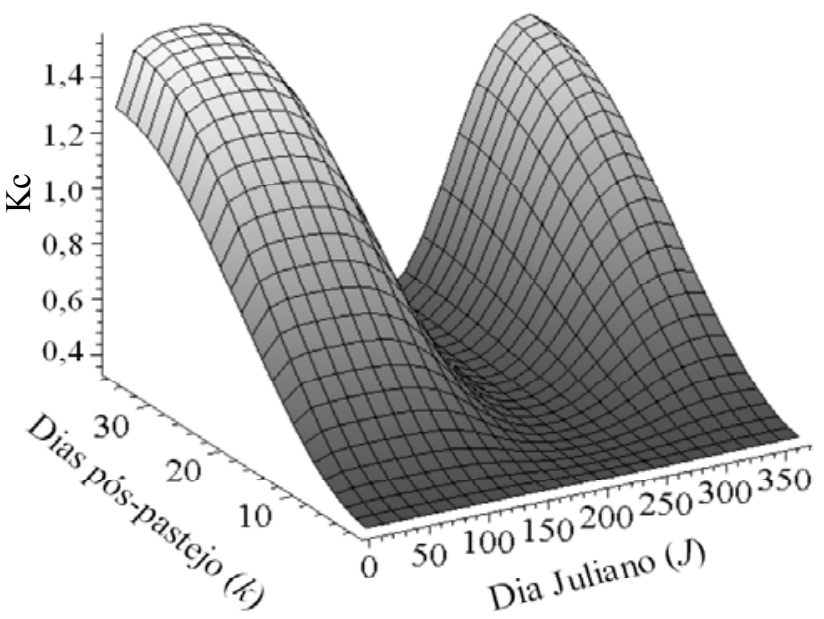

B.

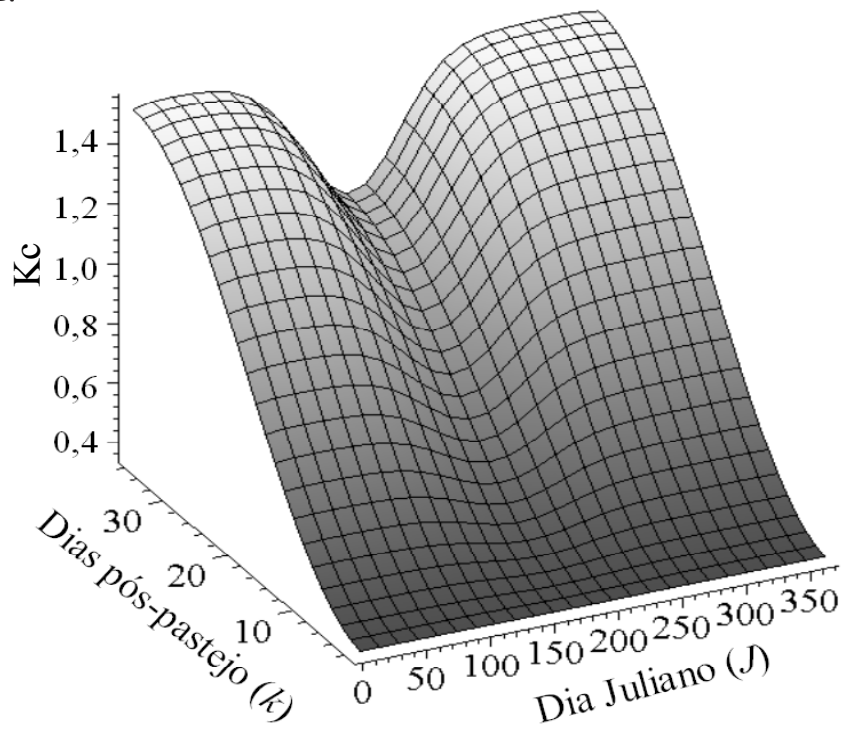

Figura 5. Kc calculado a partir do modelo senoidal em pastagem com ciclo completo de 36 dias durante um ano para as regiões de Piracicaba (A) e Pereira Barreto (B) conforme observações de campo (Lourenço et al, 2001); notase, também, que os valores de Kc no início e no final de um ciclo serão os mesmos para os dois modelos em cada região, cuja diferença estará apenas na taxa de variação do Kc.

\section{Lâminas de irrigação (Estudo de caso)}

Na simulação dos dias de descanso de cada parcela tem-se que os animais se encontram em pastejo na parcela 6 , enquanto a parcela com maior quantidade de dias de descanso é a seguinte (parcela 7) (Tabela 2). Os $\mathrm{Kc}_{\max }$ s calculados por meio do modelo logístico para Piracicaba e Pereira Barreto, foram de 1,52 e 1,54 para fevereiro $(\mathrm{J}=32)$, e de 0,42 e 1,30 para agosto $(\mathrm{J}=213)$, respectivamente. Observa-se, mais uma vez, que o $\mathrm{Kc}_{\text {max }}$ de agosto é baixo para Piracicaba $(0,42)$, resultado da baixa quantidade de energia disponível no período.

A Figura 6 apresenta a lâmina de irrigação pela Eq. 5 , com Kc obtido pelos modelos linear e senoidal para a região de Piracicaba, em fevereiro. Como o cálculo da lâmina se dá de maneira discreta, não se observa variação significativa entre as estimativas a partir dos dois modelos, mas que o modelo linear apresenta o mesmo padrão de variação do modelo senoidal.

$\mathrm{Na}$ simulação para as épocas de fevereiro e agosto e se considerando a região de Piracicaba, o modelo mostrou-se sensível à capacidade de desenvolvimento de cada período, aqui relacionada com a $\mathrm{UF}_{36}$ (Figura 7A). A temperatura média observada no mês em que a cultura esteve em desenvolvimento (julho) é de $17,9^{\circ} \mathrm{C}$, enquanto em janeiro é de $24,4^{\circ} \mathrm{C}$, implicando em que o Kc em agosto variou apenas de 0,35 a 0,42 (modelo

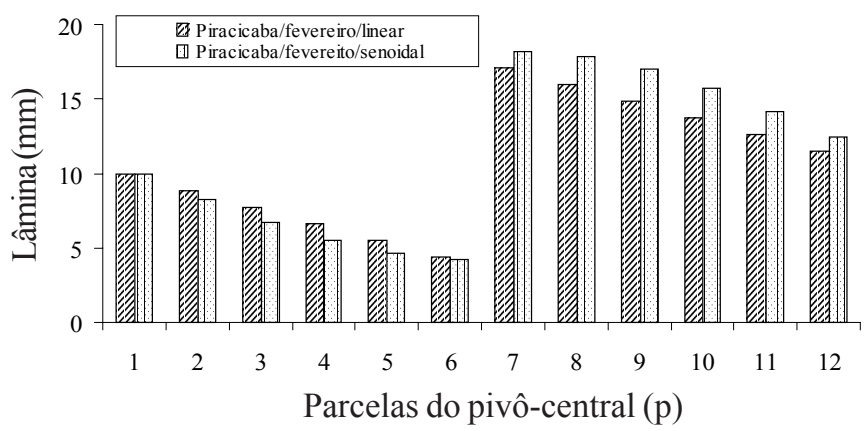

Figura 6. Simulação da lâmina de irrigação para a região de Piracicaba para o mês de fevereiro, utilizando-se os modelos linear e senoidal

Tabela 2. Dados gerais de dias de descanso para cada parcela do pivô-central e seus respectivos valores de Kc, calculados para cada região/época/modelo

\begin{tabular}{|c|c|c|c|c|c|c|c|c|c|c|c|c|c|}
\hline \multirow{2}{*}{ Região } & \multirow{2}{*}{ Modelo/Mês } & \multicolumn{12}{|c|}{ Parcela (Dias de descanso)* } \\
\hline & & $1(16)$ & $2(13)$ & $3(10)$ & $4(7)$ & $5(4)$ & $6(1)$ & $7(35)$ & $8(32)$ & $9(29)$ & $10(26)$ & $11(23)$ & $12(20)$ \\
\hline \multirow{4}{*}{ Piracicaba } & linear/fevereiro & 0,87 & 0,77 & 0,68 & 0,58 & 0,48 & 0,38 & 1,49 & 1,39 & 1,29 & 1,20 & 1,10 & 1,00 \\
\hline & senoidal/fevereiro & 0,83 & 0,69 & 0,56 & 0,46 & 0,39 & 0,35 & 1,52 & 1,48 & 1,41 & 1,31 & 1,18 & 1,04 \\
\hline & linear/agosto & 0,38 & 0,38 & 0,37 & 0,36 & 0,36 & 0,35 & 0,42 & 0,41 & 0,41 & 0,40 & 0,39 & 0,39 \\
\hline & senoidal/agosto & 0,38 & 0,37 & 0,36 & 0,36 & 0,35 & 0,35 & 0,42 & 0,42 & 0,41 & 0,41 & 0,40 & 0,39 \\
\hline \multirow{4}{*}{$\begin{array}{l}\text { Pereira } \\
\text { Barreto }\end{array}$} & linear/fevereiro & 0,88 & 0,78 & 0,68 & 0,58 & 0,48 & 0,38 & 1,51 & 1,41 & 1,31 & 1,21 & 1,11 & 1,01 \\
\hline & senoidal/fevereiro & 0,84 & 0,69 & 0,56 & 0,46 & 0,39 & 0,35 & 1,54 & 1,50 & 1,43 & 1,33 & 1,20 & 1,05 \\
\hline & linear/agosto & 0,77 & 0,69 & 0,61 & 0,53 & 0,46 & 0,38 & 1,27 & 1,19 & 1,12 & 1,04 & 0,96 & 0,88 \\
\hline & senoidal/agosto & 0,74 & 0,62 & 0,52 & 0,44 & 0,38 & 0,35 & 1,30 & 1,27 & 1,21 & 1,13 & 1,03 & 0,91 \\
\hline
\end{tabular}

* Numero entre parentese refere-se dias de descanso da referida parcela 
senoidal) enquanto em fevereiro o Kc variou de 0,35 a 1,54 durante o ciclo total da pastagem; também, é bastante clara a sensibilidade do modelo quando foram consideradas as duas regiões para o mês de agosto (Figura 7B). A região de Pereira Barreto, apesar de também se encontrar em um mês de inverno, apresenta temperaturas médias mais elevadas que a região de Piracicaba para este período $\left(21,2\right.$ contra $\left.17,9^{\circ} \mathrm{C}\right)$, refletindo na maior variabilidade das lâminas; ainda para Piracicaba, como há pouco desenvolvimento para parcelas com maior ou menor número de dias de descanso, a tendência é se aplicar uma lâmina bastante próxima com aquela calculada na parcela de controle $(10 \mathrm{~mm})$.

A.

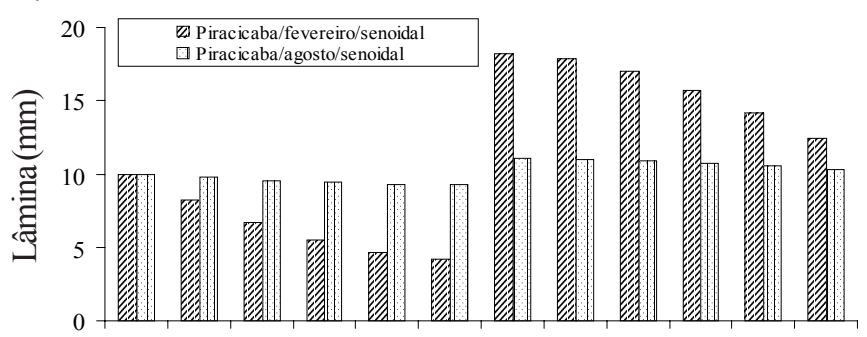

B.

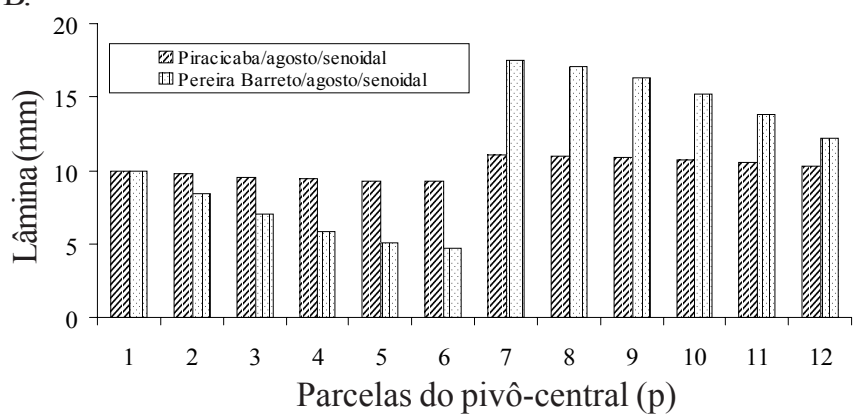

Figura 7. Lâminas de irrigação referentes aos meses de fevereiro e agosto para Piracicaba (A); e para o mês de agosto para Piracicaba e Pereira Barreto (B) utilizando-se o modelo senoidal

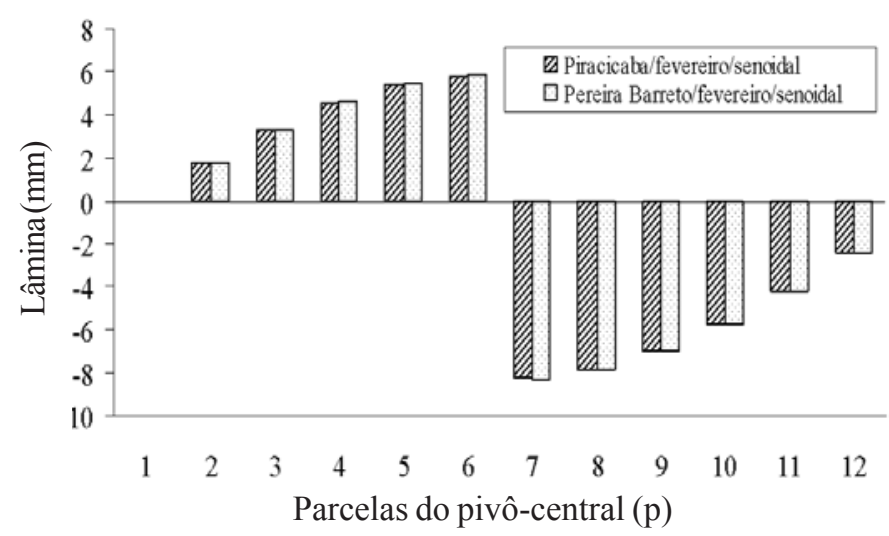

Figura 8. Diferença entre lâmina de $10 \mathrm{~mm}$ aplicada em todo o pivô-central com a aplicação de lâmina diferenciada segundo o modelo proposto
A Figura 8 representa a diferença entre uma lâmina de 10 mm que seria aplicada em toda a área do pivô, desconsiderandose a variação de desenvolvimento da pastagem entre as parcelas, com a estimativa de lâmina a irrigar realizada neste trabalho, para as regiões de Piracicaba e Pereira Barreto. Observa-se que nas primeiras parcelas haveria excesso de água, enquanto a partir da sétima parcela (inclusive) ocorreria um déficit de água em ambas as regiões prejudicando, assim, o desenvolvimento da pastagem, além de um desperdício de água e energia.

\section{CONCLUSÃO}

Foi apresentado, neste trabalho, um modelo para cálculo de lâminas de irrigação em pastagem irrigada sob pivô-central que, teoricamente, respondeu de maneira coerente, tanto no que diz respeito à aplicação de lâminas distintas de irrigação de acordo com o estádio de desenvolvimento da parcela como, também, à capacidade de desenvolvimento da pastagem de determinada região e período, baseada na unidade fototérmica.

\section{LITERATURA CITADA}

LES-ESALQ/USP. Base de dados agroclimáticos Piracicaba, SP. http://ce.esalq.usp.br/bhbrasil/Saopaulo/. 15 de Nov. de 2002.

Lourenço, L.F.; Coelho, R.D.; Soria, L.G.T.; Pinheiro, V.D.; Corsi, M. Coeficiente de cultura (Kc) do capim Tanzânia (Panicum maximum Jacq) irrigado por pivô central. In: Reunião Anual da Sociedade Brasileira de Zootecnia, 38, 2001, Piracicaba. Anais. Piracicaba: SBZ, 2001. p.1013-1017.

Mello, A.C.L.; Lourenço, L.F.; Medeiros, H.R.; Pedreira, C.G.S.; Corsi, M. Dinâmica do índice de área foliar de capim Tanzânia em função do fotoperíodo e da temperatura do ar. In: Fisiologia de plantas no novo milênio: desafios e perspectivas, 2001, Ilhéus. Anais. Congresso Brasileiro de Fisiologia Vegetal, 8. Ilhéus, 2001. p. 130.

Müller, M.S.; Fancelli, A.L.; Dourado Neto, D.; Garcia, A.G.; Ovejero, R.F.L. Produtividade do Panicum maximum cv. Mombaça irrigado, sob pastejo rotacionado. Scientia Agrícola, Piracicaba, v.59, p.427-433, 2002.

van Genuchten, M. T. A closed form equation for predicting the hydraulic conductivity of unsaturated soils. Soil Science Society America Journal, Madison, v.44, p.892-898, 1980.

Villa Nova, N.A.; Carreteiro, M.V.; Scardua, R. Um modelo de avaliação do crescimento de cana-de-açúcar (Saccharum spp.) em termos da ação combinada do fotoperíodo e da temperatura média do ar. In: Congresso Brasileiro de Agrometeorologia, 2, 1983, Campinas. Anais. Campinas: Sociedade Brasileira de Agrometeorologia, 1983. p.31-48. 